\title{
Duodenal Carcinoid with Cystic Hepatic Metastasis Presenting with Severe Anemia and Heart Failure
}

\author{
Article by Chenna Keshava BG ${ }^{1}$, KNN Anand ${ }^{2}$, Ashwin Kulkarni ${ }^{3}$, Anil Kumar T ${ }^{4}$, \\ ${ }^{1}$ Consultant Intensivist, ${ }^{2}$ Post graduate student Dept. Of Medicine, ${ }^{3}$ Asst. Professor, \\ ${ }^{4}$ Professor of Medicine, M.S. Ramaiah Medical College Bangalore, Karnataka, India \\ E-mail: ${ }^{1}$ ckeshava7@gmail.com
}

\begin{abstract}
Introduction: Carcinoid tumours are neoplasms of neuroendocrine cells with varying symptoms and malignant potential. Cystic hepatic metastasis secondary to carcinoids is a rare entity. There are very few reports in literature (around four as of date) and all have been in relation to ileal carcinoid or post chemotherapy. Most of the carcinoids present with diarrhoea, flushing, pruritis. Severe anemia as presenting symptom is rare.

Case report: Herein we describe a 66 year old gentleman who presented with severe anemia and heart failure. An OGD (Oesophago gastro duodenal endoscopy) done as part of anemia work-up revealed multiple, duodenal, centrally umbilicated nodules. Histopathology and special stains (chromogranin \& synaptophysin) confirmed it to be Grade 1 Neuroendocrine tumour (carcinoid) with a Ki-67 index of less than 2\%. CECT abdomen showed two small cystic lesions, the largest one measuring $2 x 3 \mathrm{cms}$. Anemia was corrected and patient improved symptomatically. He was placed on conservative management for Neuroendocrine tumours and is on regular follow up.

Discussion: In this case patient presented with severe anemia and heart failure. He had no other symptoms like flushing, diarrhoea. The rarity of this case is the presence of cystic hepatic metastasis and presentation with severe anemia. This is the first case reported in which a duodenal carcinoid is diagnosed during the workup for anemia. Here another interesting and rare feature is the presence of cystic hepatic metastasis which is quite unusual.

Case report: A 66 year old male, known case of CVA not on treatment, hypertensive on treatment admitted with history of progressive easy fatigability, progressive bilateral lower limb swelling, decreased appetite for 15 days. Patient denied history of diarrhea, vomiting, melena or hematochezia, diabetes, hypertension or cardiac problems .Physical examination revealed pallor, bilateral pitting pedal oedema, raised JVP, with pansystolic murmur at tricuspid area, S3 with bilateral basal crepitations and rhonchi with tender right hypochondrium. His initial workup revealed hemoglobin of $4.3 \mathrm{gm} / \mathrm{dl}$ and peripheral blood smear showed microcytic hypochromic anaemia. Liver and kidney functions were within normal limits with a serum albumin of $3.4 \mathrm{mg} / \mathrm{dl}$. $2 \mathrm{D}$ ECHO revealed dilated $R A, R V$ with a severe tricuspid regurgitation (TR) with PASP of $70 \mathrm{mmHg}$. LVEF 56\%,No RWMA, mild MR \& AR. Oesophago-gastro duodenoscopy(OGD) revealed multiple duodenal nodules with central umbilication in D1,D2,D3 with largest measuring $2 \times 3 \mathrm{~cm}$ (?Carcinoid) and was biopsied for histopathological examination(HPE) (fig 1). Contrast enhanced computerised tomography(CE-CT) thorax and abdomen revealed enlarged liver $(15.5 \mathrm{~cm})$ with two well defined non enhancing cystic lesions in segments $4 A$ of liver (measuring $36 \times 31 \times 32 \mathrm{~mm}$ and $8 \times 9 \times 4 \mathrm{~mm}$ ) and pyloric and duodenal wall thickening suggesting a possibility neoplastic etiology(fig 2). HPE (Fig 3) revealed well differentiated neuroendocrine tumor (WHO grade1) with EMA, synaptophysin, chromogranin positive and a Ki-67 index: of 2\%. Patient transfused with three pint of packed RBC and was symptomatically better, In view of the low grade of the NETs patient is on regular follow up
\end{abstract}

\section{Discussion}

Neuroendocrine tumors (NETs), also known as Carcinoids, originally Karzinoide in 1907; (=Cancer like, not the preferred terminology anymore except in case of NETs of lung and thymus) are a heterogeneous group of relatively rare neoplasms arising from the diffuse neuroendocrine system. The clinical and pathologic features resemble both the organ of origin and neuroendocrine neoplasms. WHO (2010) has classified (Table 1) NETs based on site of origin, clinical syndrome and 
differentiation(1). They are classified as functional and non-functional, based on their secreted products. Nearly two-thirds are functional NETs and the classical example is carcinoid tumors. The other examples of functional NETs is insulinomas, gastrinomas, VIPomas (vasoactive intestinal peptide), glucagonomas and somatostatinomas (2). The classical midgut NETs was referred to as carcinoid which is now referred to as neuroendocrine tumors. The diagnosis is reliant on either positive histology, or elevated 24-hour urinary 5-HIAA levels. The latter test has a sensitivity of $75 \%$ and a specificity of up to $100 \%$, provided that the use of certain drugs and foods are excluded. Most NETs express synaptophysin (a synaptic vesicle glycoprotein present in neuroendocrine cells) and chromogranin-A by immune-histochemistry. Chromogranin-A by contrast, is a nonspecific marker, and is often raised in patients with a carcinoid tumor. Depending on the cut-off values and assays used, its sensitivity and specificity are $75 \%$ and $84 \%$, respectively.(3,4).

NETs can arise anywhere in the body but the most common site of origin is the gastrointestinal tract (GIT-67.5\%), since it is the largest reservoir of neuroendocrine cells followed by the bronchopulmonary system (25.3\%). In the GIT most of them occur in the small intestine (41.8\%), rectum(27.4\%), and stomach(8.7\%). These tumors have a female preponderance and most frequently seen in fifth to seventh decades. NETs may secrete as many as forty secretory products (vasoactive substances), the most prominent being 5-hydroxytryptamine (5-HT=serotonin). These tumor products are usually inactivated by the liver but when the production exceeds the capacity for degradation, the classical carcinoid syndrome (CS) ensues (10-15\% of all carcinoids). The triad of CS is cutaneous flushing, bronchospasm with wheezing and. It is a well known fact that only one third of carcinoids present with the typical triad (5). Most patients with NETs have metastatic disease at diagnosis, with regional or distant metastasis observed in $50 \%$ of patients (6). Duodenal tumors $<1 \mathrm{~cm}$ rarely metastasize, whereas $33 \%$ of those $>2 \mathrm{~cm}$ do. Liver metastases are usually solid and vascular which on CT and magnetic resonance (MR) scans are accentuated with contrast. Primarily cystic appearance is exceedingly rare in untreated duodenal NETs and all the reports are in association with ileal NETs.(7) Cystic changes due to central tumor necrosis, are described in hepatic metastases after chemotherapeutic treatment.

Carcinoid heart disease (CHD-Hedinger syndrome) is a rare and unique manifestation seen in up to $60 \%$ of patients with NETs, typically inducing abnormalities of the right side of the heart. CHD is associated most frequently with NETs originating in the small bowel (72\%). A slight male preponderance(60\%) has been reported, with a mean age at diagnosis of 56-63 years.(8)

Our patient being a male patient, in his late sixties presented with features of severe anemia (hb4.4gm\%), bilateral pitting pedal edema, severe TR and weight loss. The TR was not explained by his co-morbidities and was presumed to be related to the carcinoid. It could have been a mild TR secondary to carcinoid accentuated by the severe anemia. A urinary HIAA level was not done as the invasive workup confirmed the diagnoses in a very short time.

In conclusion, carcinoid known for its varied sites of origin can be confusing, but a high index of suspicion with an early HPE helps in confirming the diagnoses and early plan of care. Based on this report we can safely conclude that carcinoid should be considered in differential diagnoses of all anemias.

1. Klimstra D, Modlin I, Coppola D, Lloyd R, Suster S. The Pathologic Classification of Neuroendocrine Tumors. Pancreas. 2010; 39(6):707-712.

2. National Comprehensive Cancer Network. NCCN clinical practice guidelines in oncology: neuroendocrine tumors.http://www.nccn.org/professionals/physician gls/f guidelines.asp. Accessed August 9, 2011.

3. Wiedenmann, B., Franke, W., Kuhn, C., Moll, R. and Gould, V. (1986). Synaptophysin: a marker protein for neuroendocrine cells and neoplasms. Proceedings of the National Academy of Sciences, 83(10), pp.3500-3504.

4. Ma, J. (2014). Detection of serum chromogranin A for diagnosis and evaluation of prognosis of gastrointestinal and pancreatic neuroendocrine tumors. World Chinese Journal of Digestology, 22(28), p.4352.

5. Modlin I, Oberg K, Chung D, Jensen R, de Herder W, Thakker R et al. Gastroenteropancreatic neuroendocrine tumours. The Lancet Oncology. 2008; 9(1):61-72. 
6. Yao JC, Hassan M, Phan A et al. One hundred years after 'carcinoid': epidemiology of and prognostic factors for neuroendocrine tumors in 35 825cases in the United States. J Clin Oncol 2008; 26: 3063-3072.

7. Fiori S, Del Gobbo A, Gaudioso G, Caccamo L, Massironi S, Cavalcoli F et al. Hepatic pseudocystic metastasis of well-differentiated ileal neuroendocrine tumor: a case report with review of the literature. Diagnostic Pathology. 2013; 8(1):148.

8. Grozinsky-Glasberg S, Grossman A, Gross D. Carcinoid Heart Disease: From Pathophysiology to Treatment - Something in the Way It Moves'. Neuroendocrinology. 2015;101(4):263-273.

Table 1. Who 2010 Classification of Carcinoid

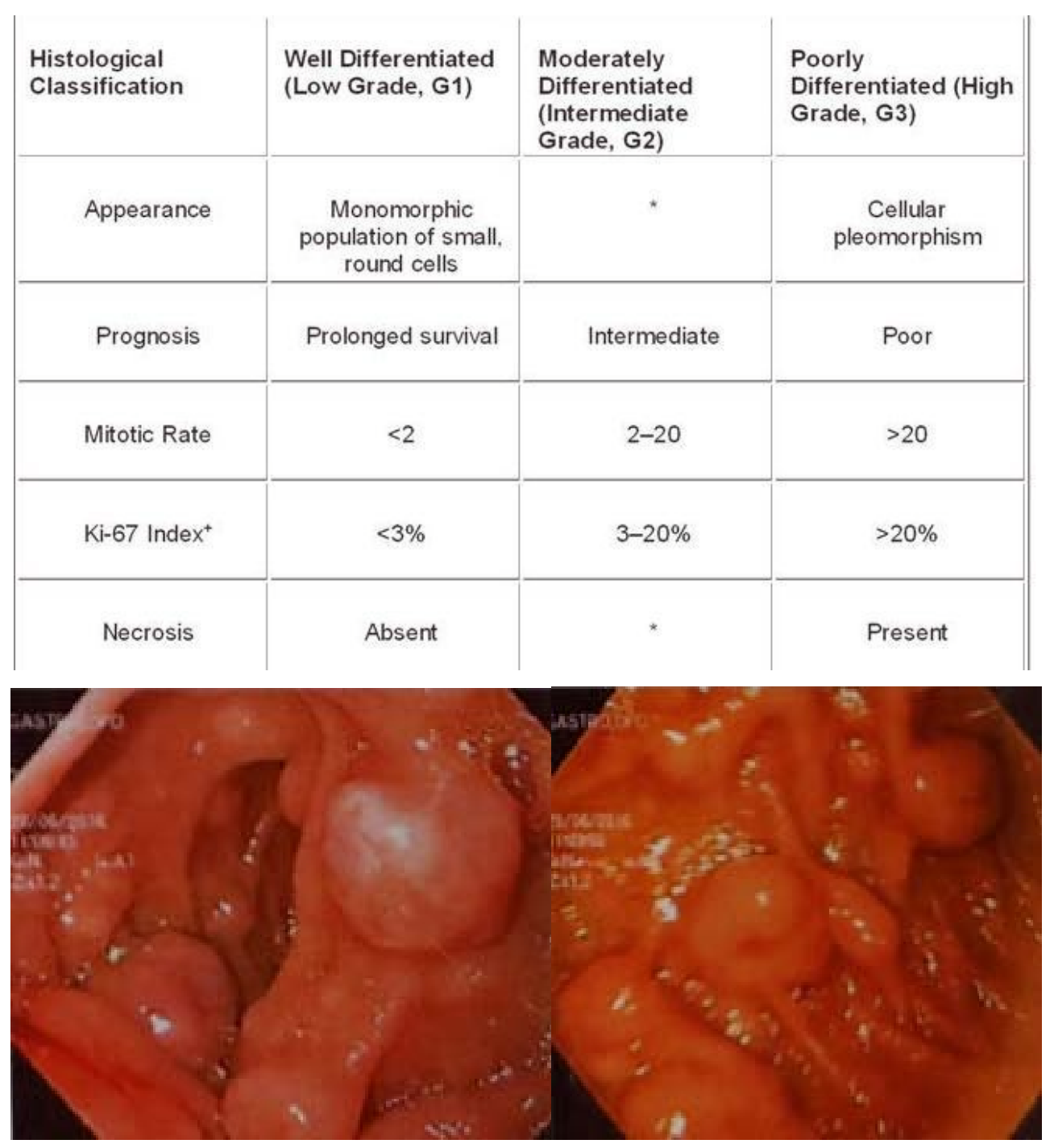

Fig 1. Multiple duodenal nodules with central umbilication in D1, D2 D3. 
Texila International Journal of Medicine

Volume 4, Issue 2, Dec 2016

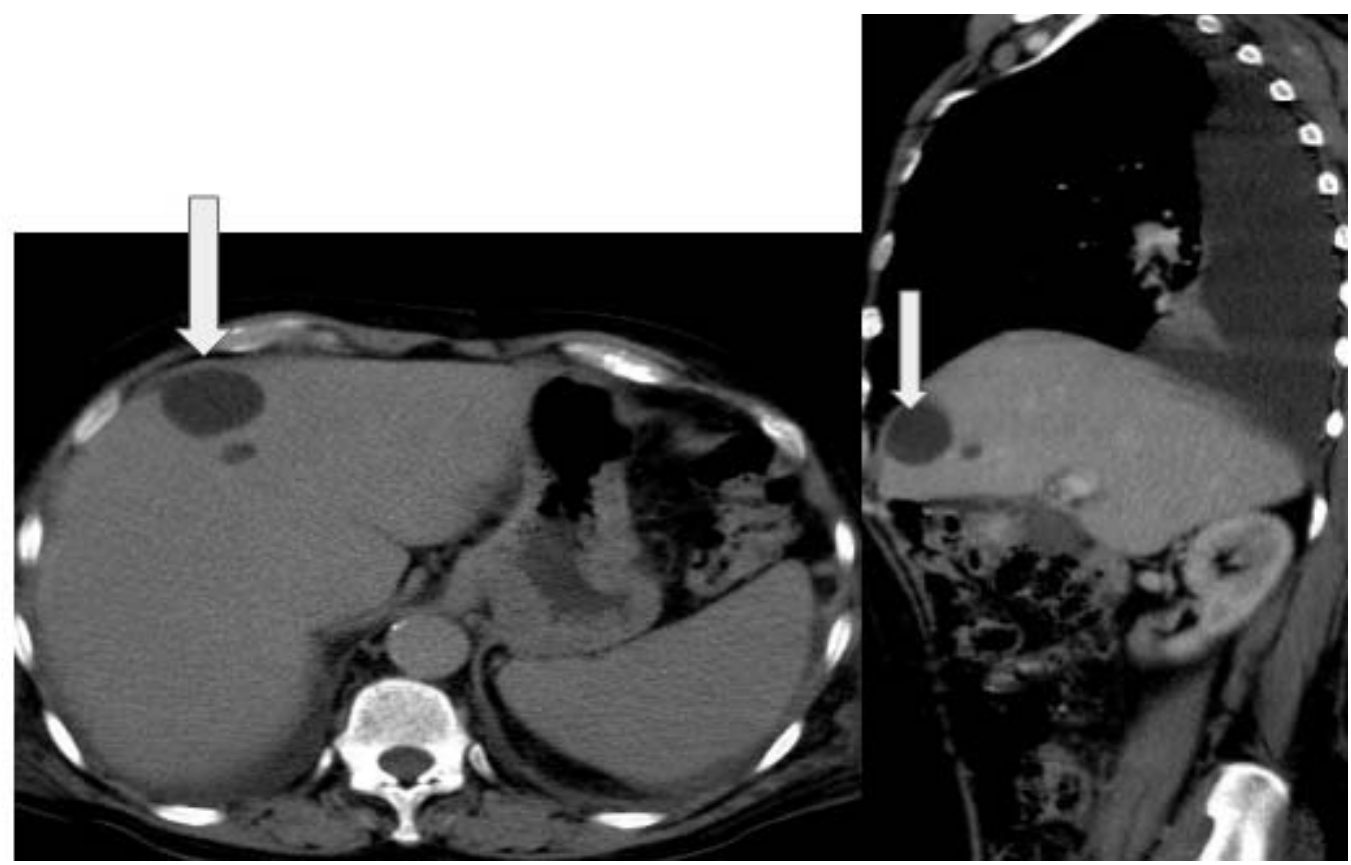

Fig 2. Transverse and longitudinal CT showing cystic lesions

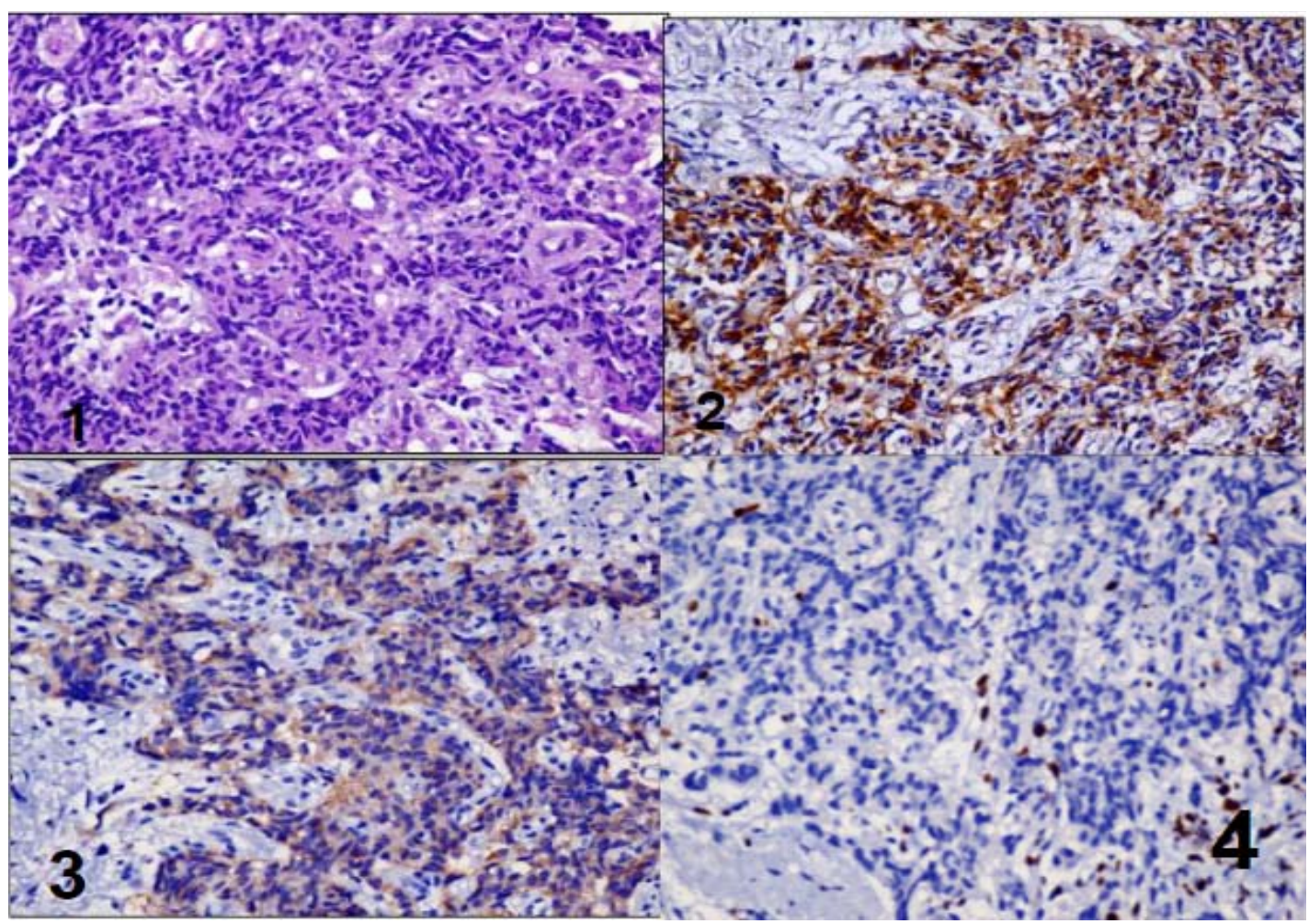

Figure 3. H\&E stain showed glands, trabecula and small solid nests of medium-sized polygonal cells with rather uniform nuclei and eosinophilic granular cytoplasm (a-c). IHC stain showed the tumor cells with positive reaction to chromogranin (2). Synaptophysin positive (3) and Ki 67 index $<2 \%$ (4). 